\title{
Single-Case Design Evaluation of Compression Therapy for Edema of the Stroke-Affected Hand
}

\author{
Louise Gustafsson, Alexandra Walter, Kylie Bower, \\ Adrienne Slaughter, Melanie Hoyle
}

MeSH TERMS

- compression bandages

- edema

- stroke

- treatment outcome

- upper extremity
Louise Gustafsson, PhD, BOccThy(Hons), is Head of Occupational Therapy, School of Health and Rehabilitation Sciences, The University of Queensland, St. Lucia, Queensland 4072, Australia; I.gustafsson@uq.edu.au

\section{Alexandra Walter, B0ccThy(Hons), was} Undergraduate Honors Student, School of Health and Rehabilitation Sciences, The University of Queensland, St. Lucia, Queensland, Australia, at the time of the study.

Kylie Bower, BOccThy(Hons), is Senior Occupational Therapist, Princess Alexandra Hospital, Metro South Health and Hospitals District, Woolloongabba, Queensland, Australia.

Adrienne Slaughter, BOccThy, is Occupational Therapist, Princess Alexandra Hospital, Metro South Health and Hospitals District, Woolloongabba, Queensland, Australia.

Melanie Hoyle, MOccThySt, PostGradDipPsych, GradDipHIthSc, is Associate Lecturer, School of Health and Rehabilitation Sciences, The University of Queensland, St. Lucia, Queensland, Australia.
OBJECTIVE. To explore the efficacy of low- and high-stretch compression bandaging for edema management in the stroke-affected upper limb.

METHOD. A single-case, ABA-design study was conducted with 8 participants alternately allocated to receive low- or high-stretch bandaging. Edema was measured with circumferential tape at four specified points from the hand to the mid-forearm. All measurements were represented graphically for visual analysis, and celeration lines were calculated to indicate the degree of slope in each phase.

RESULTS. Visual analysis indicated fluctuating edema volume in the first baseline phase, decreasing edema volume in the intervention phase, and increasing edema volume in the second baseline phase. The results did not clearly distinguish between the two bandaging groups.

CONCLUSION. Compression bandaging may have benefits in the management of edema after stroke. Further research is required to identify factors contributing to the long-term maintenance of reductions gained after compression bandaging.

Gustafsson, L., Walter, A., Bower, K., Slaughter, A., \& Hoyle, M. (2014). Single-case design evaluation of compression therapy for edema of the stroke-affected hand. American Journal of Occupational Therapy, 68, 203-211. http://dx. doi.org/10.5014/ajot.2014.009423

nnually, 15 million people worldwide have a stroke (Mackay \& Mensah, A2004). Of those 15 million people, one-third will be permanently disabled, placing the responsibility for their care on families and communities. As the population continues to age, the burden of stroke is projected to rise from 38 million disability-affected life years in 1990 to 61 million by the year 2020 (Mackay \& Mensah, 2004). Edema of the hemiplegic upper limb is a condition often encountered by medical professionals working in the rehabilitation of clients after a stroke (Giudice, 1990). Edema has been linked to a combination of dependency, immobility, and impaired lymphatic function and venous return (Roper, Redford, \& Tallis, 1999). If left unmanaged, edema can lead to significant decreases in upper-limb function resulting in a reduced capacity to complete tasks of daily living and thus affecting quality of life (Faghri, 1997). Therefore, the management of edema is an important role of the health professional involved in stroke management.

A survey of clinicians (Gustafsson \& Yates, 2009) has identified that the interventions commonly used for the management of edema did not align with the evidence-based statements for edema from the Evidence-Based Review of Stroke Rehabilitation (EBRSR) from Canada (Foley, Teasell, Jutai, Bhogal, \& Kruger, 2007) and the Australian National Stroke Foundation (NSF; 2010) Guidelines for Stroke Management. Specifically, clinicians reported using a combination of management techniques such as elevation, active movement, massage, and compression bandaging (Gustafsson \& Yates, 2009) that are often discussed as possible management for edema after injury (Villeco, 2012). The 
contrast between the evidence-based statements and clinical practice may not be indicative of poor clinical practice but rather of the need to further develop the evidence base for the interventions commonly applied. Therefore, the purpose of this study was to investigate the efficacy of compression bandaging, a commonly used intervention technique that currently has limited research evidence for use with poststroke edema.

\section{Etiology of Poststroke Edema}

Poststroke edema has been attributed to impaired functioning of the lymphatic system and venous return as a result of immobility and dependency; however, the exact etiology remains unclear (Dirette \& Hinojosa, 1994; ExtonSmith \& Crockett, 1957; Faghri, 1997). The physiology of poststroke edema is therefore distinctly different from the edema present in the local inflammatory processes of injury or surgery (Villeco, 2012). People with severe muscle weakness are particularly at risk of developing persistent edema because of the inability to produce muscular contractions that would normally assist in lymphatic flow (Faghri, 1997). Lymphatic flow also varies with the position of the limb in relation to the heart; elevation increases flow, and dependency inhibits the movement of lymph in the vessels (Exton-Smith \& Crockett, 1957; Warlow et al., 1979). Evidence has supported that although a certain amount of edema is reversible, persistent edema can lead to fibrosis of the tissue planes, resulting in longterm upper-limb impairment (Villeco, Hunter, \& Mackin, 2002). This possibility reinforces the importance of early management of edema in clinical practice.

\section{Evidence for Management}

Several excellent clinical practice guidelines are available for the management of stroke, including the U.S. Department of Veterans Affairs, U.S. Department of Defense, and American Heart Association/American Stroke Association (2010) practice guideline, the American Heart Foundation Scientific Statement (Miller et al., 2010), the EBRSR (Foley et al., 2007) from the Canadian group, and the guidelines from the Australian NSF (2010). The guidelines from the U.S. groups do not include statements specific to the management of edema and are therefore not included in this introduction to the current evidence for management of edema. The clinical practice guidelines created by the EBRSR and the NSF include recommendations for the management of edema in the hemiplegic upper limb based on the findings of a small number of studies. Edema is not an area of current research focus and has limited new evidence, despite its impact on recovery. The transferability of current research results is difficult because of study limitations, small sample sizes, and the absence of supporting evidence in independent studies. Both guidelines conclude that the evidence that electrical stimulation (ES; Faghri, 1997) and continuous passive motion (CPM; Giudice, 1990) reduce hand edema is limited.

The evidence for the application of ES is based on the outcomes of a repeated-measures randomized controlled trial investigating the efficacy of ES to induce contraction in the wrist and finger flexors and extensors of participants $(n=8)$ less than 6 mo poststroke (Faghri, 1997). This study's results demonstrated that $30 \mathrm{~min}$ of ES was significantly more effective in reducing hand edema than 30 min of elevation alone. Although included in the guidelines, reductions gained after $30 \mathrm{~min}$ of ES returned to pretreatment levels within $24 \mathrm{hr}$, and further investigations into the long-term effects of ES were not carried out. Studies of ES are ongoing, but few are concentrated on edema as a primary outcome.

CPM is recommended on the basis of the results of a multisite, crossover design study conducted to investigate the hypothesis that $30 \mathrm{~min}$ of CPM of the digits in combination with limb elevation would result in a significantly larger reduction of hand edema than elevation alone (Giudice, 1990). Measurements of participants' hand volume, finger circumference, and finger stiffness were recorded after a stroke $(n=11)$. All measurements failed to meet the required level for statistical significance. The effect sizes calculated for the measurements were large $(r>.50)$, indicating a high degree of difference between the two treatments and suggesting that a larger sample size would have reached a significant level. No subsequent or recent studies of this intervention for edema have been reported.

The NSF guidelines also recommended the inclusion of dynamic pressure garments to reduce edema with a low level of evidence based on a single crossover case-control study (Gracies et al., 2000). Gracies and colleagues (2000) assessed the efficacy of tailored upper-limb spandex garments, designed as dynamic splints, on multiple factors including swelling. Of the participants with swelling $(n=6)$, results indicated a slight but significant reduction in circumference at both the fingers and the forearm when the dynamic splint was worn for a 3-hr period. Because of the combination of the study's short duration (2 days), participants' limited length of time in the garment $(3 \mathrm{hr})$, small sample size, and nonhomogeneous participants, the study's results did not indicate long-term benefits from the garment. This recommendation was not included in the EBRSR (Foley et al., 2007) because of these factors. 


\section{Clinical Practice}

The clinical implementation of the research evidence for edema management was investigated as one component of a survey of Australian occupational therapists $(n=55$; Gustafsson \& Yates, 2009). Although the study has several limitations, including a self-selection bias, a small sample size, and fixed responses, the findings identified an inconsistent application of the evidence in clinical practice. Interventions reportedly used included ES, pressure garments, compression bandaging, elevation, early active exercise, dynamic spandex splints, and retrograde massage. The most commonly reported interventions were elevation and early active exercise; ES was incorporated into treatment less frequently than other interventions despite evidence supporting its efficacy. Compression bandaging was reported as an intervention technique used clinically $53 \%$ of the time at the elbow and $58 \%$ at the wrist (Gustafsson \& Yates, 2009). This technique, although prevalent in clinical practice, has no existing research evidence and is the basis of this study.

\section{Compression Bandaging}

The physiological rationale for bandaging is based on its ability to exert force on the tissues, thus preserving and strengthening tissue pressure and facilitating venous and lymphatic flow (Casley-Smith \& Casley-Smith, 1997; Villeco, 2012). Evidence supporting efficacy has been reported among the population with lymphedema (Badger, Peacock, \& Mortimer, 2000; Damstra \& Partsch, 2009; Johansson, Albertsson, Ingvar, \& Ekdahl, 1999) but not in the population who have had a stroke. The two categories of bandaging clinically used in compression therapy are high and low stretch. High-stretch bandages have a high resting pressure and low working pressure and are commonly used for acute edema (Johansson et al., 1999; Villeco, 2012). Low-stretch bandages have a low resting pressure and high working pressure and are more commonly used in the treatment of chronic edema because they increase the total tissue pressure when the muscle contracts, thus compressing the lymphatic system and facilitating tissue drainage (Johansson et al., 1999; Villeco, 2012). Currently, no evidence exists as to whether one is more effective than the other in reducing edema in the stroke population.

\section{Current Study}

Few empirical studies have been published investigating edema management in the hemiplegic upper limb, and as a result, clinicians frequently use interventions that do not have a firm evidence base and are often based on evidence from alternate areas of edema management. This study contributes to the current body of evidence for edema management by exploring the efficacy of compression bandaging, specifically investigating two types of commonly used bandages, low stretch and high stretch. This study is one in a series of studies to inform development of a larger research trial.

\section{Method}

\section{Research Design}

Ethical approval to conduct a single-case study using an ABA design was granted by the Princess Alexandra Hospital and the University of Queensland ethics committees in Queensland, Australia. Informed written consent was obtained from all participants.

\section{Participants}

Participants were recruited from a major metropolitan hospital. For inclusion in the study, participants had to be medically stable, have cognitive and communicative abilities to consent, and have clinically evident edema present in their affected upper limb. Clinically evident edema was defined as observable swelling compared with the nonaffected upper limb. Participants were excluded from the study if the treating doctor declined medical consent on the basis of any vascular or medical conditions for which compression bandaging was contraindicated. Once consent was provided, the participant was included in the study and alternately allocated to either the low- or high-stretch group. Allocation of the first participant to low-stretch bandaging was randomly assigned, and alternate allocation occurred for subsequent participants.

\section{Instrumentation}

To ensure reliable measurements of limb circumference, a standard protocol was developed. A circumferential measure was taken at four specific points on the affected upper limb: mid-middle phalanx of the third digit, the metacarpophalangeal joints, the wrist at the ulnar styloid, and the mid-forearm $(10 \mathrm{~cm}$ above the ulnar styloid) using an edema measurement tape. All measurements were taken at the same time of day because edema volume can vary from morning to evening, regardless of treatment (Faghri, 1997). The measurements were taken by three individual members of the research team. Circumferential measurements taken by a consistent and trained experimenter following a standard protocol have been shown to have high levels of intrarater reliability (Jansen et al., 2010; Lewis, 2010).

To further establish interrater reliability, the experimenters measured six volunteers at the four specified 
points. Cronbach's $\alpha$ was calculated for each individual measurement point and was .982 at the mid-middle phalanx of the third digit, .962 at the metacarpophalangeal, .980 at the wrist, and .990 at the forearm. These values exceed the minimal acceptable values for interrater reliability (Bravo \& Potvin, 1991).

\section{Intervention}

Three researchers followed a standard procedure based on specialized training when applying compression bandaging. Circumferential compression bandaging was applied moving distally from individual fingers to more proximally covering the hand and wrist, following the technique commonly applied in the clinical setting (Casley-Smith \& Casley-Smith, 1997; Földi \& Földi, 2006). This technique involves spiraling the bandages along the length of the fingers and covering the dorsal and palmar aspects of the hand with $50 \%$ overlap. Participants 1, 3, 5, and 7 received the low-stretch Handy ${ }^{\mathrm{TM}}$ Gauze Cohesive bandages (Smith \& Nephew, Mount Waverly, Victoria, Australia), and Participants 2, 4, 6, and 8 received the high-stretch Coban ${ }^{\mathrm{TM}}$ bandages (3M, North Ryde, New South Wales, Australia).

\section{Procedure}

Initial Baseline $A^{1}$. At recruitment to the study, the edema in the participants' affected upper limb was recorded for 3 consecutive days. Standard therapy continued and may have included edema interventions such as elevation and massage. Compression bandaging was applied to the affected hand after the third baseline measurement.

Intervention Phase $B$. The participants wore the compression bandaging for a total of 1 wk. During the week, the bandaging was removed on three occasions to allow circumferential measurements to be recorded. The bandages were reapplied after the first two measurements but not after the third measurement, which was taken on the final day of the intervention phase.

Final Baseline $A^{2}$. The final baseline phase replicated the measurement protocol for the intervention phase. That is, three circumferential measurements were taken during the 1 -wk period.

\section{Data Analysis}

Data for individual participants were plotted on a graph for statistical and visual analysis (Ottenbacher, 1986; Portney \& Watkins, 2000). Each graph presented the circumferential measurements at each anatomical position over time for a single participant. Visual analyses were conducted by identifying changes within and between phases. Data within a phase were analyzed according to stability and trends or directions of change. Data between phases were analyzed according to changes in levels, trends, and slopes. The third measurement point served as a final baseline measure but also as the first measurement point for the intervention phase, which was demarcated on the graph with a vertical line. The sixth measurement point was also demarcated by a vertical line to indicate the final measurement for the intervention phase and the first measurement point for the second baseline phase.

To assist with visual analysis, a celeration line was constructed in each phase to represent the linear trend and slope for the data series (Portney \& Watkins, 2000). In this method, data in each phase were split in half along the $x$ axis. These halves were then halved again, and the median values were found. The celeration line was drawn connecting the medians of each quarter of the data. The celeration line was then used to estimate the rate of change in each phase. The slope was calculated by taking two $y$ values on two points of the line and dividing the numerically larger number by the smaller number. By looking at the direction of the celeration line, the direction of the rate of change was determined (Portney $\&$ Watkins, 2000).

\section{Results}

Eight participants were eligible for inclusion in this study, and all participants who were approached provided informed consent. All 8 eligible participants completed the study. Demographic and medical information were gathered from participants and medical charts; these characteristics are summarized in Table 1.

The values of individual celeration lines are reported in Table 2. The graphs of representative participant data are shown in Figures 1 and 2. Participant 6 (Figure 1) was discharged from hospital before the final measurement point; thus, eight rather than nine measurement points are illustrated.

Table 1. Background Patient Characteristics

\begin{tabular}{|c|c|c|c|c|c|c|c|c|}
\hline \multirow[b]{2}{*}{ Characteristic } & \multicolumn{4}{|c|}{ Low-Stretch Group } & \multicolumn{4}{|c|}{ High-Stretch Group } \\
\hline & $\mathrm{P} 1$ & P3 & P5 & P7 & $\mathrm{P} 2$ & P4 & P6 & P8 \\
\hline Days from stroke & 36 & 56 & 56 & 21 & 25 & 117 & 59 & 67 \\
\hline Affected limb & Left & Right & Right & Left & Right & Left & Left & Left \\
\hline Type of edema & Soft & Soft & Soft & Soft & Soft & Soft & Pitting & Soft \\
\hline Splinting & Yes & Yes & Yes & No & No & Yes & No & Yes \\
\hline Hypertonicity & Yes & Yes & No & No & No & Yes & Yes & No \\
\hline Active movement & No & No & Yes & No & Limited & No & No & No \\
\hline
\end{tabular}

Note. $\mathrm{P}=$ participant. 


\begin{tabular}{|c|c|c|c|c|c|c|c|c|}
\hline \multirow[b]{2}{*}{ Measurement Point } & \multicolumn{4}{|c|}{ Low-Stretch Group } & \multicolumn{4}{|c|}{ High-Stretch Group } \\
\hline & P1 & P3 & P5 & P7 & P2 & P4 & P6 & P8 \\
\hline \multicolumn{9}{|c|}{ Proximal phalanx 3rd digit } \\
\hline Intervention & -1.048 & -1.025 & -1.026 & -1.034 & -1.046 & -1.014 & -1.137 & -1.125 \\
\hline Baseline & +1.063 & +1.025 & +1.034 & +1.034 & +1.400 & +1.022 & +1.161 & +1.011 \\
\hline \multicolumn{9}{|l|}{ MCP } \\
\hline Intervention & -1.003 & -1.024 & -1.029 & -1.025 & -1.012 & +1.009 & -1.079 & -1.015 \\
\hline Baseline & +1.063 & +1.029 & +1.045 & +1.057 & +1.048 & +1.002 & +1.087 & +1.010 \\
\hline \multicolumn{9}{|l|}{ Wrist at ulnar styloid } \\
\hline Baseline & +1.012 & +1.006 & -1.006 & +1.023 & +1.026 & -1.024 & +1.021 & +1.030 \\
\hline Intervention & +1.003 & -1.003 & -1.012 & -1.007 & -1.013 & -1.012 & -1.026 & -1.037 \\
\hline Baseline & -1.054 & +1.052 & +1.012 & -1.029 & -1.054 & +1.006 & -1.021 & +1.101 \\
\hline
\end{tabular}

Note. Plus (+) and minus (-) signs indicate direction of slope. MCP = metacarpophalangeal; $\mathrm{P}=$ participant.

\section{Within-Phase Analysis}

Within-phase analysis of the data indicated variability during each independent phase. The circumference at the four measurement points appeared to fluctuate both at baseline and during the intervention and was particularly evident in the distal measurement points.

Despite fluctuations in edema volume, general trends were observed when analyzing the data within independent phases. During the baseline $A^{1}$ phase, a general trend of increasing volume was evident. In the intervention phase, two trends were evident dependent on anatomical position. Distally, edema volume was observed to consistently decrease in the intervention phase, whereas proximally edema volume was observed to increase, decrease, or remain static. During the baseline $\mathrm{A}^{2}$ phase, an increasing trend was observed among participants with regard to edema volume at the three distal measurement points.

\section{Between-Phase Analysis}

Phase $A^{1}$ : Intervention Phase. The changes in celeration line direction indicated that both low- and high-stretch compression bandages frequently reduced the edema volume. This reduction was particularly evident distally because all participants had reduced measurements in the mid-middle phalanx of the third digit and the metacarpophalangeal joint during the intervention phase. Proximally, this reduction was not as evident because three participants $(1,2$, and 7$)$ did not have reduced measurements at the mid-forearm.

Intervention Phase to Baseline $A^{2}$. The changes in celeration line direction indicated that the reductions recorded were not maintained and edema volume returned to preintervention levels.

\section{Between-Group Analysis}

The data did not clearly indicate an evident difference between the low- and high-stretch groups. Both groups had decreases in limb circumference in the intervention phase, with the celeration lines indicating similar slopes of decline.

\section{Discussion}

The aim of this study was to investigate the efficacy of two commonly used compression bandages on the management of edema in the stroke-affected hand. The between-groups phase analysis of the data indicated that compression bandaging may have benefits in the shortterm management of edema in people after a stroke. All participants displayed a reduction in edema of the upper limb over the course of the intervention phase regardless of group allocation. This finding is consistent with lymphedema evidence supporting compression bandaging as a management technique (Badger et al., 2000; Damstra \& Partsch, 2009; Johansson et al., 1999). The withingroups phase analysis of the data indicated that the nature of edema is variable, with fluctuations in fluid volume evident across all four anatomical positions measured. Robust conclusions regarding the effects of the intervention are limited by these fluctuations and the inability to clearly establish a stable baseline period. However, it seems reasonable to infer that compression bandaging does reduce 


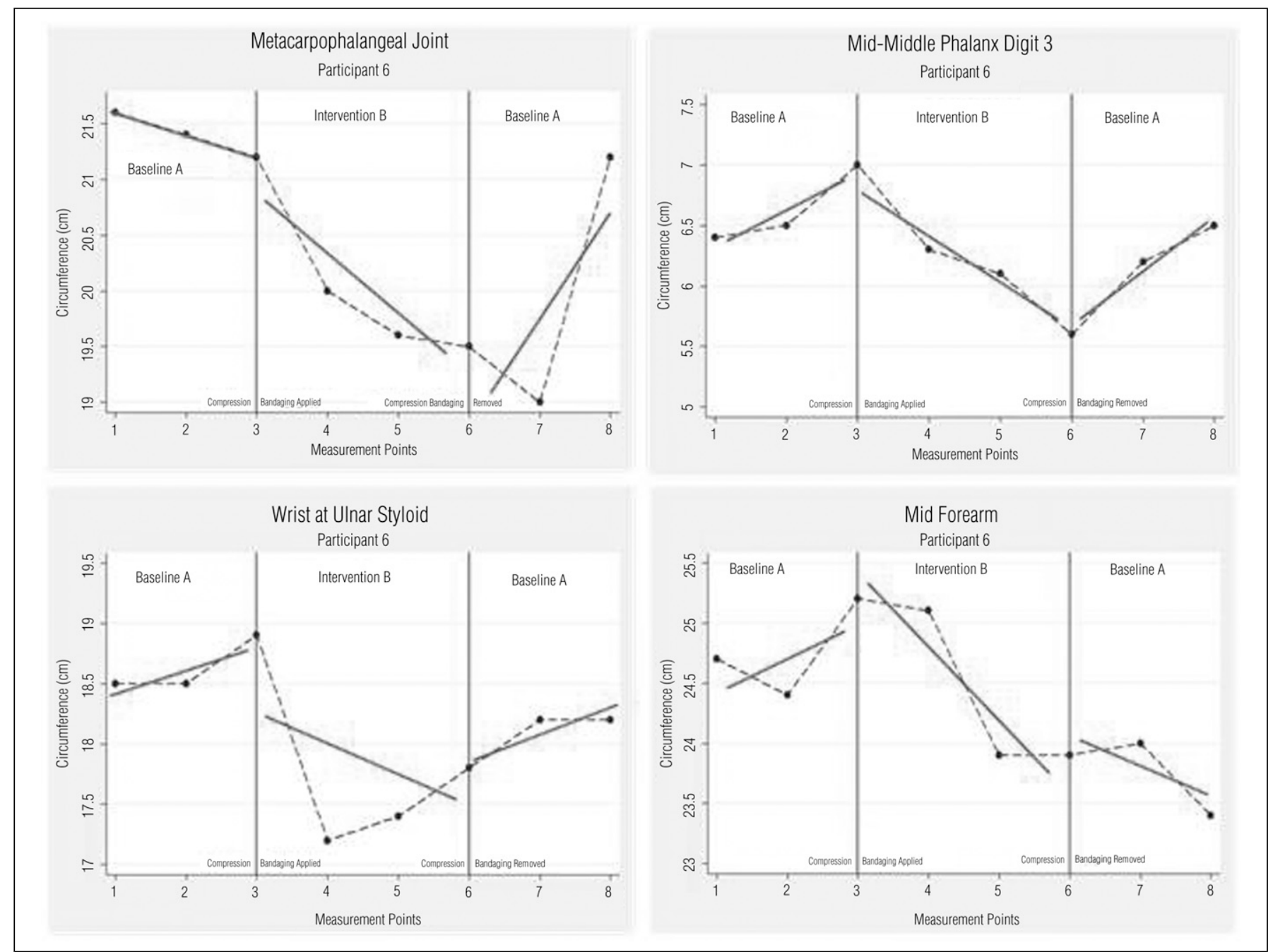

Figure 1. Circumferential measurements of Participant 6, high-stretch bandaging applied. The figure presents the circumferential measurements at four anatomical positions with high-stretch bandages applied. The dashed line indicates the raw data, and the solid line represents the celeration line.

edema, on the basis of trends in the circumferential measurements between adjacent phases. The results have the potential to support clinicians in their clinical reasoning when selecting compression bandaging as an adjunct to the recommendations in the EBRSR (Foley et al., 2007) and the NSF (2010) guidelines.

The within-phase fluctuations in edema volume may be the result of several interacting factors including content and timing of therapy activities, positioning, and limb movement near the time of measurement. Movement within a day may affect measurements by altering the muscular activity in the upper limb. Several studies have found that passive compression from muscular contraction augments the propulsions of lymph toward the heart by compressing the adjacent lymphatic vessels and veins (Giudice, 1990; Vasudevan \& Melvin, 1979). In addition, the literature has established that positioning affects the amount of edema in the upper limb because it can either assist or inhibit the flow of fluid (Exton-Smith \& Crockett,
1957; Warlow et al., 1979). A participant whose upper limb has been in a position of dependency before measurement will likely record larger measurements because the lymphatic drainage system has been inhibited and fluid has pooled in the upper limb, for example, if it has been hanging down while mobilizing. Conversely, edema measurements will be lower if the participant's limb has been elevated, facilitating the return of fluid toward the heart. Optimal positioning may be difficult to maintain when clients have reduced cognition, perception, and staff supervision.

Movement of edematous fluid was evident when analyzing the trends between phases of individual participants at the four measurement points. Although volumetric methods are frequently documented as the gold standard for measuring volume of the limb (Karges, Mark, Stikeleather, \& Worrell, 2003), circumferential measurements were crucial in identifying movement of fluid. Changes in the circumferential measurements indicated 


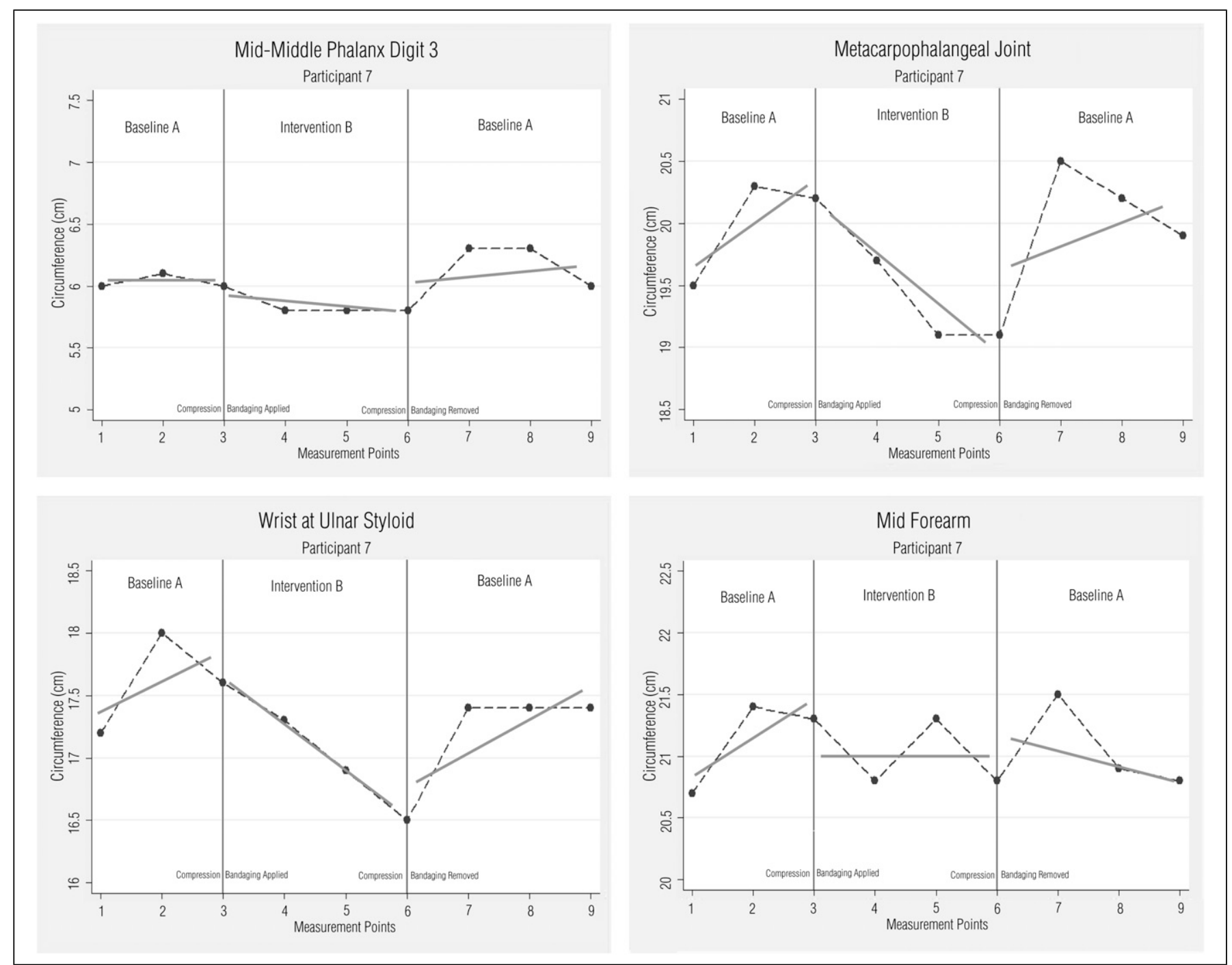

Figure 2. Circumferential measurements of Participant 7, low-stretch bandaging applied. The figure presents the circumferential measurements at four anatomical positions with low-stretch bandages applied. The dashed line indicates the raw data, and the solid line represents the celeration line.

that the edema fluid was pushed proximally during the intervention phase as the compression bandages increased the tissue pressure. This observation was indicated by decreased measurements at the fingers, relative stability at the hand and wrist, and a trend for increasing measurements at the forearm. As the compression was removed and tissue pressure subsequently decreased, the fluid returned distally to the hand, indicated by increased measurements at the fingers, hand, and wrist and a trend for reducing measurements at the forearm. This result suggests that the application of compression bandages in isolation did not have a lasting effect on the reduction of the edema in the hand.

The limited long-term effect of compression bandaging is also indicated by the between-groups phase analysis of the intervention phase and the second baseline phase. It is evident that the edema returned to pretreatment levels or higher in several participants, namely at the metacarpophalangeal joints and wrist. This result indicates that compression bandaging did not exert a significant effect on the underlying mechanisms causing edema in the affected upper limb. As acknowledged previously, the physiological processes causing edema after a stroke are unknown, although they have been linked to changes in venous and lymphatic function as a result of immobility and dependency (Dirette \& Hinojosa, 1994; Exton-Smith \& Crockett, 1957; Faghri, 1997; Villeco et al., 2002; Warlow et al., 1979). This study indicates that compression bandaging is unable to alter these factors, and a maintenance strategy is required to preserve reductions gained.

It is hypothesized that three primary factors may contribute to the long-term maintenance of reductions gained after compression bandaging. First, increasing the surface area of the compression bandaging to extend to the shoulder may potentially improve outcomes because fluid is pushed into the axillary lymph nodes and returned to the 
vascular system. In keeping with clinical practice at the facility involved in this study, compression bandaging was applied only to the level of the wrist. Second, the application of a maintenance device, such as a compression garment, may contribute to preventing the return of edema fluid because the tissue pressure is maintained. As previously mentioned, the study by Gracies and colleagues (2000) suggested support for the use of dynamic compression garments; however, because of this study's limitations further investigation is required. The application of a maintenance device such as a compression garment was not implemented in this study. Finally, increasing muscle activity and movement will likely have a positive effect on minimizing edema long term (Giudice, 1990; Vasudevan \& Melvin, 1979). If clinicians are able to maximize motor recovery, it is likely to minimize longterm edema.

\section{Limitations and Future Research}

The ability to make robust conclusions is limited by the single case-study research design. Future research should aim to use larger samples of participants and implement stratified random sampling to control for possible influencing factors and individual variance, thereby facilitating greater transferability of results. Additionally, it would be highly beneficial for future research to investigate the most effective bandaging regimen, which may include investigating the efficacy of bandaging to different anatomical points along the arm (e.g., to the wrist vs. to the axilla) and the efficacy of different maintenance devices. In combination, the results would provide evidence to support clinicians in the implementation of a compression bandaging regimen that facilitates long-term reductions in edema volume.

\section{Conclusion and Implications for Clinical Practice}

The results of this study suggest that compression bandaging is an effective intervention technique for the reduction of edema in the stroke-affected upper limb. This finding supports clinical practice that has reported the application of compression bandaging despite limited research evidence. However, many factors remain unclear, and this study has highlighted the fluctuating and individual nature of edema in the hemiplegic upper limb. An ongoing program of research is required to determine the most effective intervention regimen with compression bandaging, including the type of bandage and hand versus upper-limb bandaging, and to establish a maintenance strategy. Clinical implications from this study include the following:

- Compression bandaging has the potential to be used as an intervention for the management of edema in the hemiplegic upper limb.

- Further research is required to identify the most effective regimen, including maintenance of reductions gained from compression bandaging over the long term.

\section{Acknowledgments}

This work was partially supported by the National Stroke Foundation (Honours Research Grant 2011). We gratefully acknowledge the support of all clinicians and clients involved in the study.

\section{References}

Badger, C. M., Peacock, J. L., \& Mortimer, P. S. (2000). A randomized, controlled, parallel-group clinical trial comparing multilayer bandaging followed by hosiery versus hosiery alone in the treatment of patients with lymphedema of the limb. Cancer, 88, 2832-2837. http://dx. doi.org/10.1002/1097-0142(20000615)88:12<2832::AIDCNCR24>3.0.CO;2-U

Bravo, G., \& Potvin, L. (1991). Estimating the reliability of continuous measures with Cronbach's alpha or the intraclass correlation coefficient: Toward the integration of two traditions. Journal of Clinical Epidemiology, 44, 381-390. http://dx.doi.org/10.1016/0895-4356(91)90076-L

Casley-Smith, J. R., \& Casley-Smith, J. R. (1997). Modern treatment for lymphoedema (5th ed.). Adelaide, South Australia: Lymphoedema Association of Australia.

Damstra, R. J., \& Partsch, H. (2009). Compression therapy in breast cancer-related lymphedema: A randomized, controlled comparative study of relation between volume and interface pressure changes. Journal of Vascular Surgery, 49, 1256-1263. http://dx.doi.org/10.1016/j.jvs. 2008.12.018

Dirette, D., \& Hinojosa, J. (1994). Effects of continuous passive motion on the edematous hands of two persons with flaccid hemiplegia. American Journal of Occupational Therapy, 48, 403-409. http://dx.doi.org/10.5014/ajot.48.5.403

Exton-Smith, A. N., \& Crockett, D. J. (1957). Nature of oedema in paralysed limbs of hemiplegic patients. BMJ, 2, 1280-1283. http://dx.doi.org/10.1136/bmj.2.5056.1280

Faghri, P. D. (1997). The effects of neuromuscular stimulationinduced muscle contraction versus elevation on hand edema in CVA patients. Journal of Hand Therapy, 10, 29-34. http:// dx.doi.org/10.1016/S0894-1130(97)80008-7

Földi, M., \& Földi, E. (2006). Földi's textbook of lymphology: For physicians and lymphoedema therapists (2nd ed.). Munich, Germany: Elsevier.

Foley, N., Teasell, R., Jutai, J., Bhogal, S., \& Kruger, E. (2007). Upper extremity interventions: Evidence-based review of stroke rehabilitation. Retrieved August 2012 from www.ebrsr.com/ reviews_details.php?Upper-Extremity-Interventions-31 
Giudice, M. L. (1990). Effects of continuous passive motion and elevation on hand edema. American Journal of Occupational Therapy, 44, 914-921. http://dx.doi.org/10.5014/ ajot.44.10.914

Gracies, J. M., Marosszeky, J. E., Renton, R., Sandanam, J., Gandevia, S. C., \& Burke, D. (2000). Short-term effects of dynamic Lycra splints on upper limb in hemiplegic patients. Archives of Physical Medicine and Rehabilitation, 81, 1547-1555. http://dx.doi.org/10.1053/apmr.2000.16346

Gustafsson, L., \& Yates, K. (2009). Are we applying interventions with research evidence when targeting secondary complications of the stroke-affected upper limb? Australian Occupational Therapy Journal, 56, 428-435. http://dx.doi. org/10.1111/j.1440-1630.2008.00757.x

Jansen, V., Radbourne, L., Fakis, A., Bradley, M., Bure, F., \& Ellis, J. (2010). Validity, responsiveness, intra- and interrater reliability of the weighted tape measure when measuring digital circumference. Hand Therapy, 15, 31-38. http://dx.doi.org/10.1258/ht.2010.010009

Johansson, K., Albertsson, M., Ingvar, C., \& Ekdahl, C. (1999). Effects of compression bandaging with or without manual lymph drainage treatment in patients with postoperative arm lymphedema. Lymphology, 32, 103-110.

Karges, J. R., Mark, B. E., Stikeleather, S. J., \& Worrell, T. W. (2003). Concurrent validity of upper-extremity volume estimates: Comparison of calculated volume derived from girth measurements and water displacement volume. Physical Therapy, 83, 134-145.

Lewis, E. S. (2010). Finger circumference measurements: Interand intra-rater reliability. Hand Therapy, 15, 69-72. http:// dx.doi.org/10.1258/ht.2010.010017

Mackay, J., \& Mensah, G. (2004). The atlas of heart disease and stroke. Geneva: World Health Organization.

Miller, E. L., Murray, L., Richards, L., Zorowitz, R. D., Bakas, T., Clark, P., \& Billinger, S. A.; American Heart Association Council on Cardiovascular Nursing and the Stroke Council. (2010). Comprehensive overview of nursing and interdisciplinary rehabilitation care of the stroke patient: A scientific statement from the American Heart Association. Stroke, 41, 2402-2448. http://dx.doi.org/10.1161/STR. 0b013e3181e7512b

National Stroke Foundation. (2010). Clinical guidelines for stroke management 2010. Retrieved from www.strokefoundation. com.au

Ottenbacher, K. J. (1986). Evaluating clinical change: Strategies for occupational and physical therapists. Baltimore: Williams \& Wilkins.

Portney, L. G., \& Watkins, M. P. (2000). Foundations of clinical research (2nd ed.). Upper Saddle River, NJ: Prentice Hall Health.

Roper, T. A., Redford, S., \& Tallis, R. C. (1999). Intermittent compression for the treatment of the oedematous hand in hemiplegic stroke: A randomized controlled trial. Age and Ageing, 28, 9-13. http://dx.doi.org/10.1093/ageing/28.1.9

U.S. Department of Veteran Affairs, U.S. Department of Defense, \& American Heart Association/American Stroke Association. (2010). VA/DoD practice guideline for the management of stroke rehabilitation. Retrieved from www. healthquality.va.gov/stroke/stroke_full_221.pdf

Vasudevan, S. V., \& Melvin, J. L. (1979). Upper extremity edema control: Rationale of the techniques. American Journal of Occupational Therapy, 33, 520-523.

Villeco, J. P. (2012). Edema: A silent but important factor. Journal of Hand Therapy, 25, 153-161, quiz 162. http:// dx.doi.org/10.1016/j.jht.2011.09.008

Villeco, J. P., Hunter, J. M., \& Mackin, E. J. (2002). Edema: Therapist's management. In E. J. Mackin, A. D. Callahan, T. M. Skirven, L. H. Schneider, \& A. L. Osterman (Eds.), Rehabilitation of the hand and upper extremity (5th ed., pp. 1026-1034). St. Louis, MO: Mosby.

Warlow, C. P., Dennis, M. S., Van Gijn, J., Sandercock, P. A. G., Bamford, J. M., \& Wardlow, J. M. (1979). Stroke: A practical guide to management. Oxford, England: Blackwell Science. 
Reproduced with permission of the copyright owner. Further reproduction prohibited without permission. 